\title{
Endoscopic Management of Esophageal Stenosis in Children with Congenital Esophageal Atresia
}

\author{
Deganello Saccomani Marco $^{1 *}$, Bortolotti Valentina ${ }^{1}$, Di Lorenzo Martina Chiara ${ }^{1}$, Gastaldi Andrea ${ }^{1}$, Cerofolini \\ Angelo², Catalano Filippo ${ }^{2}$, Tomba Francesco ${ }^{2}$, Rodella Luca $^{2}$, Camoglio Francesco Saverio ${ }^{3}$, Sciré Gabriella ${ }^{3}$, \\ Piacentini Giorgio ${ }^{1}$ and Banzato Claudia ${ }^{1}$ \\ ${ }^{1}$ Department of Pediatrics, Woman's and Child's University Hospital of Verona, Italy
}

${ }^{2}$ Department of Emergency Endoscopy, University Hospital of Verona, Italy

${ }^{3}$ Department of Pediatric Surgery, Woman's and Child's University Hospital of Verona, Italy

Submission: October 01, 2020; Published: October 20, 2020

*Corresponding author: Deganello Saccomani Marco, Department of Pediatrics, Woman's and Child's University Hospital of Verona, Italy

Abstract

Background and Study Aim: Esophageal stenosis (ES) is the most common complication associated with congenital esophageal atresia (EA). There is no consensus regarding the endoscopic management of strictures in terms of timing and techniques of dilation. The aim of this study is to describe the endoscopic approach of esophageal stenosis in children with EA admitted to our tertiary care center.

Patients and Methods: A retrospective descriptive single center study was conducted. Data of all patients diagnosed with EA admitted to Woman's and Child's University Hospital of Verona, Italy, between 2004 and 2017, were reviewed and collected. For each patient type of EA, associated malformations, age of surgical correction, number and timing of dilations, technique of dilation and side effects of the procedure were registered.

Results: Thirty-seven patients with EA were admitted to our center between 2004 and 2017. Twenty of them were excluded from the analysis for insufficient data. Five of the seventeen patients enrolled were affected by VACTERL syndrome and 3 had other congenital malformations. Twelve (70 \%) subjects had tracheoesophageal fistula, all of them with type C EA. Three (18\%) children presented with long gap EA. All patients underwent surgical correction within 2 months of life and an endoscopic control was performed in all of them. Eleven patients (65\%), 8 with Type C EA and 3 with Type A EA, underwent endoscopic dilations because of ES. Eight of them (72\%) needed more than one dilation due to anastomotic re- stenosis. Median age of first dilation was 3 months (1-12 months), whereas median age of last dilation was 6 months (1-18 months). Only in one case was used balloon dilator, whereas all other procedures were performed using Savary- Gilliard semirigid dilators. One patient (Type C EA with long gap) underwent surgical re-treatment due to an endoscopic complication (fistula recurrence). Six of the seventeen subjects (35\%) enrolled developed long-term complications (stridor; severe esophagitis; subglottic stenosis; esophageal diverticulum; dysphagia).

Conclusion: Our data confirmed that anastomotic stricture is frequent in patients with EA after surgical correction. Endoscopic management of stenosis is a safe and effective procedure that lead to a limited number of complications. Regular follow-up by a multidisciplinary team is fundamental to prevent and treat short-term and long-term complications.

Keywords: Esophageal atresia; Esophageal stenosis; Esophageal dilation; Pediatric endoscopy; Children

Abbreviations: ES: Esophageal Stenosis; EA: Esophageal Atresia; TEF: Tracheoesophageal Fistula; VACTERL: Vertebral Defects, Anal Atresia, Cardiac Defects, Tracheoesophageal Fistula, Renal and Limb Anomalies; GERD: Gastroesophageal Reflux Disease; ESPGHAN: European Society for Paediatric Gastroenterology, Hepatology and Nutrition; ESGE: European Society of Gastrointestinal Endoscopy; NASPGHAN: North American Society for Pediatric Gastroenterology, Hepatology and Nutrition

\section{Introduction}

\section{Background}

Esophageal atresia (EA) is the most common congenital malformation of the esophagus, occurring in 1 in 2500 to 4500 newborns [1]. EA is characterized by an interruption of the esophageal lumen, with or without a connection with the trachea (tracheoesophageal fistula) [2]. Several anatomical variants are described according to Gross classification: isolated EA without 
fistula (Gross A, 11\%); EA with proximal tracheoesophageal fistula (TEF) (Gross B, 2\%); EA with distal TEF (Gross C, 86\%); EA with proximal and distal TEF (Gross D, <1\%); tracheoesophageal fistula without atresia (Gross E, 4\%) [2]. Although EA may present as an isolated abnormality, in approximately $50 \%$ of cases one or multiple associated defects can be identified. The most common of them is a congenital heart disease [3]. No specific genetic abnormalities have been detected in EA, but, nevertheless, deletions $22 \mathrm{q} 11$ or $17 \mathrm{q} 22 \mathrm{q} 23.3$ and trisomy for chromosomes 18 or 21 have been associated with a greater risk of developing EA. Furthermore, EA may occur as part of VACTERL association (at least three features between: Vertebral defects, Anal atresia, Cardiac defects, Tracheoesophageal fistula, Renal and Limb anomalies) [4].

Despite EA is usually diagnosed within the first 24 hours of life, it may also be suspected during pregnancy in presence of polyhydramnios and absence of gastric bubble on ultrasound scan. The clinical picture of EA is characterized by drooling, choking during feeding or recurrent episodes of cyanosis and dyspnoea. The inability to pass a gastric tube into the stomach support the suspect of atresia. The diagnosis of EA is usually confirmed by chest X-ray, using an orogastric catheter or air as contrast to recognize the proximal pouch $[2,5]$.

Once the diagnosis of EA has been established, a surgical approach is necessary to repair the defect. The surgical technique consists in the closure of the TEF and in a circular end-toend anastomosis of the proximal and distal segments of the esophagus. In case of long gap between the two ends, defined as a gap of 2 or more centimeters or vertebral spaces, the primary anastomosis may be delayed [6]. Although the treatment of EA has a high survival rate, several complications may occur early or late after surgical repair [7]. Early complications may include anastomotic leakage, anastomotic stricture and recurrence of TEF, whereas gastroesophageal reflux, dysmotility and tracheomalacia are frequent late complications [2].

Esophageal stricture (ES) is the most common complication of EA repair, occurring from 18-60\% of cases [1]. The ES is defined as a narrowing at the level of the anastomosis associated with clinically relevant symptoms such as feeding difficulties, vomits and coughing during feeds. Food impaction, recurrent respiratory infections, and failure to thrive are the main complications related to ES. However, there are no symptoms or signs enough sensitive or specific to predict the severity of the stricture $[8,9]$. Predisposing factors for ES are long-gap EA with consequent tension on the suture line, postoperative anastomotic leak, and gastroesophageal reflux disease (GERD) [10].

The recommended fist-line treatment for ES is endoscopic dilation, whose primary aim is to achieve symptom relief [10]. However, there is no consensus regarding timing and technique of dilations [8]. Several studies reported no relevant differences in outcomes between routine and symptomatic ES treatment [7].
Therefore, to reduce the risk of dilation-related complications (perforation, bleeding, recurrence of fistula and infection), endoscopic dilations should be performed only in symptomatic patients $[7,9]$. However, in children with repaired EA at high risk of strictures (long gap EA, post-anastomotic leak) many authors suggest routine dilations to prevent ES complications even if asymptomatic [10].

Endoscopic dilation may be performed either with balloon or Savary dilators. The hydro-static balloon, expanded by the injection of liquid, applies only a radial force on the strictures [7]. On the other hand, Savary dilators going through the stricture, exert a radial and a longitudinal force. The bougies are tapered tubes with fixed diameter ranging from 5 to $14 \mathrm{~mm}$ and the dilation is obtained increasing dilator diameter $[10,11]$. Several studies showed that both techniques are equally safe and effective, despite there are no randomized controlled trials in children with EA comparing the two techniques. Therefore, the choice of dilation technique should be based on operator experience $[10,12]$

Once the diameter of the esophagus has been restored, unfortunately, in some patients, the stenosis may recur. The last ESPGHAN - ESGE Guidelines (European Society for Paediatric Gastroenterology, Hepatology and Nutrition - European Society of Gastrointestinal Endoscopy) on diagnostic and therapeutic endoscopy defines refractory stricture the "inability to successfully remediate the anatomic problem to obtain ageappropriate feeding possibilities after a maximum of 5 dilation sessions with maximal 4-week intervals"; whereas a recurrent stenosis is the "inability to maintain a satisfactory luminal diameter for 4 weeks once the age-appropriate feeding diameter has been achieved" [13]. Refractory and recurrent stenosis are the main challenge in the long-term management of patients with EA and may significantly impact the prognosis and the quality of life of these children.

Recently, the first Guidelines for the assessment and treatment of children with EA have been published by the European Society for Paediatric Gastroenterology, Hepatology and Nutrition (ESPGHAN) and the North American Society for Pediatric Gastroenterology, Hepatology and Nutrition (NASPGHAN) [8]. Although these Guidelines provide an essential support for the management of children with EA, there is still a lack of evidence regarding the endoscopic approach of anastomotic strictures in terms of timing and techniques of dilations in these patients. The aim of this study is to describe the endoscopic management of esophageal stenosis in children with EA admitted to our tertiary care center to contribute to determine the optimal treatment strategy in these patients.

\section{Methods}

A retrospective descriptive single center study was conducted. Data of all patients diagnosed with EA admitted to the Woman's and Child's University Hospital of Verona, between 2004 and 2017, 
were reviewed and collected. For each patient the following data were registered: gestational age, birth weight, prenatal diagnosis, type of EA according to Gross classification (A-B-C-D and long gap vs short gap), associated malformations or syndromes, age of surgical correction, number and timing of dilations, technique of dilations, side effects of the dilation procedure.

Diagnostic and therapeutic endoscopies were performed using Olympus $\AA^{\circledR}$ Evis Exera III CV190 video endoscope. Balloon dilators or Savary- Gilliard semi-rigid dilators were used for endoscopic dilation. The choice between the two devices was based on endoscopist's experience. In all cases a wire-guide and fluoroscopy were used during the procedures. All endoscopies were performed under general anesthesia. Considering the retrospective and observational nature of the study and the complete anonymization of data, ethics approval and consent to participate were not obtained.

\section{Results}

A total of 37 patients diagnosed with EA were admitted to our center between 2004 and 2017. Twenty patients were excluded from the analysis for insufficient data. The final sample was then composed by 17 patients, 12 males and 5 females (M: $F=2,4: 1$ ) Table 1. Preterm birth, considered as gestational age $<37$ weeks, was described in 6 subjects (35.3\%). The average birth weight was 2715 grams (range 1780-3900g). Prenatal diagnosis of EA was made in $2 / 17$ patients $(11.8 \%)$. Associated malformations were found in $8 / 17$ (47\%) children; five of them presented with VACTERL syndrome.

Table 1: Main features of subjects with EA admitted to Woman's and Child's University Hospital between 2004 and 2017.

\begin{tabular}{|c|c|c|c|c|c|c|}
\hline $\begin{array}{l}\text { Type EA (Gross } \\
\text { classification) }\end{array}$ & $\begin{array}{c}\text { Presence } \\
\text { of Long gap } \\
(*)\end{array}$ & $\begin{array}{c}\text { Endoscopic } \\
\text { Dilation } \\
\text { (number) }\end{array}$ & $\begin{array}{c}\text { Long Term Complica- } \\
\text { tions }\end{array}$ & $\begin{array}{l}\text { Birth Weight } \\
\text { (grams) }\end{array}$ & $\begin{array}{l}\text { Gestational } \\
\text { Age (weeks) }\end{array}$ & $\begin{array}{l}\text { Presence of VACTERL or } \\
\text { other Malformation }\left({ }^{*}\right)\end{array}$ \\
\hline A & - & 0 & - & Unknown & $>37$ & - \\
\hline A & - & 1 & $\begin{array}{l}\text { Type C esophagitis with } \\
\text { fundoplicatio }\end{array}$ & 1780 & 34 & - \\
\hline A & - & 0 & Dysphagia & Unknown & $>37$ & - \\
\hline A & - & 2 & - & 3900 & $>37$ & - \\
\hline A & $*$ & 3 & - & Unknown & $>37$ & $*$ \\
\hline $\mathrm{C}$ & - & 1 & - & 2330 & $>37$ & - \\
\hline $\mathrm{C}$ & - & 2 & - & 3900 & $>37$ & - \\
\hline $\mathrm{C}$ & - & 2 & - & 2550 & 35 & * \\
\hline $\mathrm{C}$ & - & 0 & subglottic stenosis & 3390 & $>37$ & $*$ \\
\hline $\mathrm{C}$ & - & 2 & - & 2820 & $>37$ & - \\
\hline $\mathrm{C}$ & - & 0 & - & 2500 & $>37$ & *(VACTERL) \\
\hline $\mathrm{C}$ & - & 0 & - & 3315 & $>37$ & *(VACTERL) \\
\hline $\mathrm{C}$ & - & 0 & $\begin{array}{l}\text { Stridor, esophageal diver- } \\
\text { ticulum }\end{array}$ & 2700 & $>37$ & - \\
\hline $\mathrm{C}$ & - & 1 & Recurrent bronchitis & 2420 & 36 & - \\
\hline $\mathrm{C}$ & $*$ & 10 & Fistula recurrence & 2335 & 34 & *(VACTERL) \\
\hline $\mathrm{C}$ & $*$ & 6 & - & 1865 & 32 & *(VACTERL) \\
\hline $\mathrm{C}$ & - & 2 & - & 2200 & 35 & *(VACTERL) \\
\hline
\end{tabular}

Twelve patients (70\%) had tracheoesophageal fistula, all with Type C EA according to Gross classification. Five subjects (30\%) had type A EA. Long gap EA, defined as a gap $>2 \mathrm{~cm}$ of length between the two esophageal pouches, was described in 3/17 cases (18\%), one with type A EA and 2 with type C EA. All patients underwent surgical correction within 2 months of life. After surgery, in all subjects an endoscopic control was performed.
Eleven children (65\%) developed an esophageal stricture at level of the anastomosis and underwent endoscopic dilation Figure 1. Eight of them had Type C EA, whereas three presented with Type A EA. The total number of dilations was 32. More than 1 dilation was needed in $8 / 11(72 \%)$ children due to re-stenosis. The median number of dilations in these patients were 2 (range $0-10)$. 


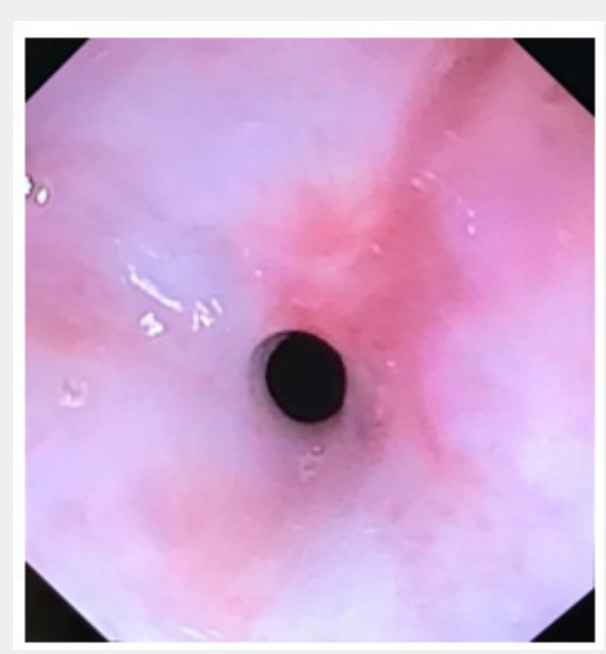

Figure 1: Severe esophageal anastomotic stenosis in a patient with long gap repaired type C EA.

In three subjects, all with long gap EA, more than 3 dilations were needed. The median age at the time of first endoscopic dilation was 3 months (range 1-12 months). The median age at the last dilation was 6 months (range 1-18 months). Only in 1 case balloon dilation was used, whereas all other procedures were performed using Savary- Gilliard semi-rigid dilators. All strictures were eventually resolved, and no patients needed surgical resection. No acute complications were observed during all dilation procedures except in one subject (type C EA with long gap) that underwent surgical re-treatment due to fistula recurrence after bougie dilation. In 6/17 (35.3\%) children were observed long-term complications such as stridor, recurrent bronchitis, severe esophagitis (fundoplication needed), subglottic stenosis, esophageal diverticulum, persistent functional dysphagia (Figure 2).

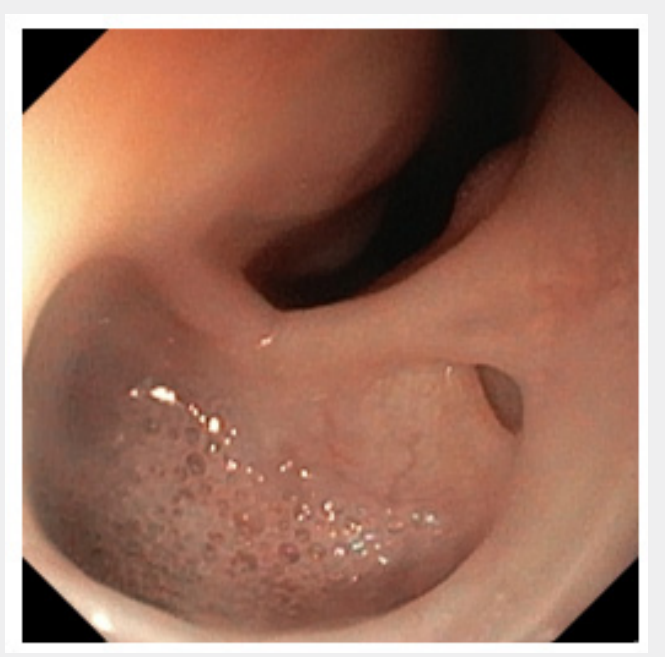

Figure 2: Large esophageal diverticulum of the anastomosis in a patient with repaired type C EA.

\section{Discussion}

Data from our experience confirm that in children with EA anastomotic stricture is a common complication, especially in subjects with long gap type C EA. Endoscopic dilation is a safe and effective treatment, but some patients may need more than one procedure. Despite the improvement of surgical technique and postoperative care, gastrointestinal and respiratory complications are still frequent, and the long-term management of these children is still challenging. In our sample, $65 \%$ of 
patients with EA developed an esophageal stricture (ES). These subjects were all affected by type A or C EA and they underwent an endoscopic dilation. As reported in the literature, our data confirm that anastomotic esophageal stricture is the most common complication after surgical repair of EA with an observed prevalence that ranges from 10 to $80 \%$ of cases $[7,8]$. As described by Kovesi et al. [3] ES is more frequent in subjects with EA type $\mathrm{A}$ and $\mathrm{C}$ rather than type $\mathrm{D}$ or $\mathrm{E}$ and about $70 \%$ of anastomotic strictures require dilation.

Endoscopic dilation is the first line of therapy for esophageal strictures [8]. All our dilations were performed with SavaryGilliard dilators except in one case in which balloon dilation was preferred. In all procedures wire-guide and fluoroscopy were used. Two retrospective studies compared the two techniques of dilation in EA patients, but results are controversial in terms of efficacy and safety $[14,15]$. According to the last ESPGHANNASPGHAN Guidelines about EA, the choice between bougie and balloon dilation should be based only on the experience of the operator. Anyway, the use of wire-guide and fluoroscopy is considered fundamental [8]. In $72 \%$ of our children more than one dilation was needed with a median of 2 dilations for each patient (range 0-10). 57\% of children with short gap EA underwent only one or two dilation procedures, whereas all patients $(100 \%)$ with long gap EA developed an anastomotic stricture that required more than 3 dilations.

As previously described in literature, gap length is an important risk factor for ES. In fact, if the distance between the two stumps of the esophagus is more than $2 \mathrm{~cm}$, after surgical repair the site of the anastomosis is under tension and the possibility of an anastomotic leak is higher [8,16-18]. Regardless of the number of procedures needed to obtain the resolution of the stenosis, the reported success rate of endoscopic dilation in literature ranged from $70 \%$ to $100 \%$ [7]. In our patients we eventually resolved all the ES with endoscopic procedures and nobody of them needed surgical stricture resection for refractory or recurrent stenosis.

In our subjects, the decision regarding the interval between dilations was based on the severity of the stricture and on the presence of symptoms. Therefore, in some patient's selective dilations were performed only when symptoms occurred, whereas in others, especially in children with long gap EA, routine dilations were planned to prevent severe re-stenosis and symptoms. In the study of Koivusalo et al. [19] the two approaches were retrospectively compared, and they concluded that dilations based on symptoms resulted in a significantly lower number of procedures with equal long-term outcomes. Furthermore, also the recent ESPGHAN-NASPGHAN Guidelines recommend a less invasive "wait-and-see approach" in which dilation should be started only when a patient becomes symptomatic. However, they also suggest a close follow-up in all children which are at high risk (long gap EA) of developing severe ES to avoid severe or complete anastomotic closure $[7,8,20]$.
A total of 32 dilations were performed in our center for ES and only $1 / 32(3,1 \%)$ endoscopic complication occurred. We observed a recurrence of fistula after the 10th dilation in a child with long gap type C EA. Surgical repair with closure of the fistula was necessary in this patient. Previous studies reported recurrence of fistula or perforation due to endoscopic dilation in $0.9-8 \%$ of procedures, mostly affecting higher risk patients $[7,21,22]$. In our sample, long-term complications were observed in one-third (35\%) of children. Stridor, recurrent bronchitis, severe esophagitis (fundoplication needed), subglottic stenosis, esophageal diverticulum and persistent functional dysphagia were described.

This finding is confirmed by the literature where is reported that motility disorders, gastro-esophageal reflux disease and respiratory symptoms are the most common long-term complications, affecting almost half of children with EA [3,18,23]. Among our patients, prematurity resulted a risk factor for longterm complications with a rate of $60 \%$ in preterm subjects versus $25 \%$ in term newborns. This data was recently reported also by Rayyan et al that described how prematurity is associated with respiratory and gastrointestinal morbidity during the first year of life and later in childhood [24]. No correlations between longterm complications and birth weight or presence of VACTERL or other associated malformations were found in our patients.

Data from our experience confirm that, despite refractory and recurrent esophageal strictures remain a major challenge, the endoscopic management of esophageal stenosis in children with EA is a safe and effective approach that lead to a limited number of complications, mostly affecting higher risk patients (long gap EA). Common shared protocols defining optimal timing of dilations and endoscopic technique are needed to improve patient's outcome. Due to the high rate of associated morbidity, which include not only gastrointestinal symptoms, patients with EA should be regularly evaluated by a multidisciplinary team, including pulmonologists and otolaryngologists, to prevent and treat promptly short-term and long-term complications.

\section{References}

1. Landisch RM, Foster S, Gregg D, Thomas Chelius, Laura D Cassidy, et al. (2017) Utilizing stricture indices to predict dilation of strictures after esophageal atresia repair. J Surg Res 216: 172-178.

2. Spitz L (2007) Oesophageal atresia. Orphanet J Rare Dis 2: 1-13.

3. Kovesi T, Rubin S (2004) Long-term complications of congenital esophageal atresia and/or tracheoesophageal fistula. Chest 126: 915925.

4. Shaw Smith C (2006) Oesophageal atresia, tracheo-oesophageal fistula, and the VACTERL association: Review of genetics and epidemiology. J Med Genet 43: 545-554.

5. Pinheiro PFM, Silva ACS, Pereira RM (2012) Current knowledge on esophageal atresia. World J Gastroenterol 18: 3662-3672.

6. Foker JE, Linden BC, Boyle EM, C Marquardt (1997) Development of 
a true primary repair for the full spectrum of esophageal atresia. Ann Surg 226: 533-543.

7. Manfredi MA (2016) Endoscopic Management of Anastomotic Esophageal Strictures Secondary to Esophageal Atresia. Gastrointest Endosc Clin N Am 26: 201-219.

8. Krishnan U, Mousa H, Dall'Oglio L, Nusrat Homaira, Rachel Rosen, et al. (2016) ESPGHAN-NASPGHAN guidelines for the evaluation and treatment of gastrointestinal and nutritional complications in children with esophageal atresia-tracheoesophageal fistula. J Pediatr Gastroenterol Nutr 63: 550-570.

9. Macchini F, Leva E, Parente G, Giorgio Farris, Valerio Gentilino, et al (2018) Classification of Esophageal Strictures following Esophageal Atresia Repair. European J Pediatr Surg 28: 243-249.

10. Tambucci R, Angelino G, De Angelis P, Filippo Torroni, Tamara Caldaro, et al. (2017) Anastomotic strictures after esophageal atresia repair: Incidence, investigations, and management, including treatment of refractory and recurrent strictures. Front Pediatr 5: 1-14.

11. Hirano I, Moy N, Heckman MG, Colleen S Thomas, Nirmala Gonsalves, et al. (2013) Endoscopic assessment of the oesophageal features of eosinophilic oesophagitis: Validation of a novel classification and grading system. Gut 62: 489-495.

12. Mark JA, Anderson BT, Pan Z, Calies Menard Katcher, Robert E Kramer (2019) Comparative analysis of adverse events after esophageal balloon and bougie dilations in children. J Pediatr Gastroenterol Nutr 68: 630-634.

13. Thomson M, Tringali A, Dumonceau JM, Marta Tavares, Merit M Tabbers, et al. (2017) Paediatric gastrointestinal endoscopy: European society for paediatric gastroenterology hepatology and nutrition and European society of gastrointestinal endoscopy guidelines. J Pediatr Gastroenterol Nutr 64: 133-153.

14. Lang T, Hümmer HP, Behrens R (2001) Balloon Dilation is Preferable to Bougienage in Children with Esophageal Atresia. Endoscopy 33: 329 335.

15. Jayakrishnan VK, Wilkinson AG (2001) Treatment of oesophageal strictures in children: A comparison of fluoroscopically guided balloon dilatation with surgical bouginage. Pediatr Radiol 31: 98-101.

16. Upadhyaya VD, Gangopadhyaya AN, Gupta DK, S P Sharma, Vijayendra Kumar, et al. (2007) Prognosis of congenital tracheoesophageal fistula with esophageal atresia on the basis of gap length. Pediatr Surg Int 23:

\section{7-771.}

17. Dall'Oglio L, Caldaro T, Foschia F, Simona Faraci, Giovanni Federici di Abriola, et al. (2016) Gastrointestinal Endoscopy: Global view Endoscopic management of esophageal stenosis in children : New and traditional treatments. World J Gastrointest Endosc 8: 212-219.

18. Castilloux J, Noble AJ, Faure C (2020) Risk Factors for Short- and LongTerm Morbidity in Children with Esophageal Atresia. J Pediatr 156: 755-760.

19. Koivusalo A, Pakarinen MP, Rintala RJ (2009) Anastomotic dilatation after repair of esophageal atresia with distal fistula. Comparison of results after routine versus selective dilatation. Dis Esophagus 22: 190-194.

20. Romeo E, Foschia F, De Angelis P, Tamara Caldaro, Giovanni Federici di Abriola, et al. (2011) Endoscopic management of congenital esophageal stenosis. J Pediatr Surg 46: 838-841.

21. Alshammari J, Quesnel S, Pierrot (2011) Endoscopic balloon dilatation of esophageal strictures in children. International J Pediatr Otorhinolarn 75: 1376-1379.

22. Poddar U, Thapa BR (2001) Benign esophageal strictures in infants and children: Results of Savary-Gilliard bougie dilation in 107 Indian children. Gastroint Endosc 54: 480-484.

23. Pedersen RN, Markow S, Kruse Andersen S, Niels Qvist, Tine Plato Hansen, et al. (2013) Esophageal Atresia: Gastroesophageal functional follow-up in 5-15 year old children. J Pediatr Surg 48: 2487-2495.

24. Rayyan M, Embrechts M, Van Veer H, Raf Aerts, Ilse Hoffman, et al. (2019) Neonatal factors predictive for respiratory and gastrointestinal morbidity after esophageal atresia repair. Pediatr Neonato 60: 261-269. 